\title{
Effects of Natural Convection from an Open Square Cavity Containing a Heated Circular Cylinder
}

\author{
Sheikh Anwar Hossain*a and M. A. Alim ${ }^{\mathrm{b}}$ \\ ${ }^{a}$ Department of Mathematics, Jagannath University, Dhaka; ${ }^{b}$ Department of Mathematics, \\ BUET, Dhaka, Bangladesh.
}

\begin{abstract}
The problem of natural convection heat transfer in a square open cavity containing a heated and conducting circular cylinder at the centre is analyzed in this paper. As boundary conditions of the cavity, the left vertical wall is kept at a constant heat flux, bottom and top wall are kept at different high and low temperature respectively. The remaining side is open. Two dimensional laminar steady state natural convection is considered. This configuration is related in the design of electronic devices, solar energy receivers, uncovered flat plate solar collectors geothermal reservoirs etc. The fluid is concerned with different Prandtl numbers, Grashof numbers and the properties of the fluid are assumed to be constant. The development of Mathematical model is governed by the coupled equations of continuity, momentum and energy and is solved by employing Galerkin weighted finite element method. Flow field and heat transfer were predicted for fluid with Pr $=$ $0.72,1.0,7.0 ; \mathrm{Gr}=10^{3}, 10^{4}, 10^{5}, 10^{6}$; inclination angles of the cavity are $\varphi=0^{\circ}, 15^{\circ}, 30^{\circ}, 45^{\circ}$ and diameter ratio $\mathrm{dr}=0.2$.The average Nusselt number increases as the increases of inclination angle of the cavity for lower Pr and lower temperature at bottom wall. The average $\mathrm{Nu}$ increases mainly for higher inclinations and for higher Gr. Various vortices and recirculations are formed into the flow field for higher Pr and higher temperature at the bottom wall.
\end{abstract}

Key words : Heat transfer, Finite element method, Natural convection, Square open cavity, Heated circular cylinder.

\section{Nomenclature:}

$C_{f x} \quad$ local skin friction coefficient

$C_{p} \quad$ specific heat at constant pressure

$F \quad$ dimensionless stream function

g gravitational acceleration $\left(\mathrm{ms}^{-2}\right)$

q Heat flux $\left(\mathrm{Wm}^{-2}\right)$

$\mathrm{dr}$ diameter ratio

$\mathrm{T}$ temperature of the fluid in the cavity $(\mathrm{K})$

$\mathrm{T}_{\mathrm{w}}$ temperature at the surface $(\mathrm{K})$

$\mathrm{Gr}$ grashof number

$\mathrm{K}$ thermal conductivity of the fluid $\left(\mathrm{Wm}^{-1} \mathrm{~K}^{-1}\right)$

$\mathrm{H}, \mathrm{L}$ height and width of the cavity (m)

D diameter of the cylinder $(\mathrm{cm})$

$\mathrm{Nu}$ Nusselt number

$\mathrm{P}$ pressure $\left(\mathrm{Nm}^{-2}\right)$

$\mathrm{P} \quad$ non-dimensional pressure

Pr Prandtl number, $v / \alpha$

$\mathrm{T}_{\mathrm{O}} \quad$ temperature of the ambient fluid (K)

$\mathrm{T}_{\mathrm{h}} \quad$ high temperature at the bottom wall (K)

$\mathrm{T}_{\mathrm{c}} \quad$ low temperature at the upper wall $(\mathrm{K})$

$\mathrm{Nu}_{\mathrm{av}}$ average Nusselt number

$\mathrm{u}, \mathrm{v}$ velocity component $\left(\mathrm{ms}^{-1}\right)$
$\mathrm{U}_{\infty} \quad$ free stream velocity $\left(\mathrm{ms}^{-1}\right)$

$\mathrm{U}_{0}$ reference velocity

$\mathrm{U}, \mathrm{V}$ non-dimensional velocity componentes

$\mathrm{X}, \mathrm{y}$ Cartesian co-ordinates (m)

$\mathrm{X}, \mathrm{Y}$ non-dimensional Cartesian co-ordinates

$\mathrm{T}_{\mathrm{cl}}$ temperature of the cylinder

\section{Introduction}

The study of natural convection in open cavities has been the subject of many experimental and numerical investigations during the past two decades. The previous researchers have investigated the effect on flow and heat transfer for different Rayleigh numbers, aspect ratios and tilt angles. As a result a number of studies have been done for natural convection heat transfer over a cylinder placed inside a closed enclosure.

Showole and Tarasuk (1993) studied experimentally and numerically, the steady state natural convection in a two dimensional isothermal open cavity. They found the experimental results for air, varying the Rayleigh number from $10^{4}$ 
to $5.510^{5}$, cavity aspect ratios of $0.25,0.5$ and 1.0 and inclination angles of $0^{\circ}, 30^{\circ}, 45^{\circ}$ and $60^{\circ}$ (for $0^{\circ}$, the wall opposite the aperture was horizontal and the angles were taken clockwise). They calculated the numerical results for Raleigh numbers between $10^{4}$ and $5.510^{5}$, inclination angles of 0 and 45 and an aspect ratio equal to one. In the result it is seen that for all Rayleigh numbers, the first inclination of the cavity caused a significant increase in the average heat transfer rate, but a further increase in the inclination angle caused very little increase in the heat transfer rate. In another result it is observed that, for $0^{\circ}$, two symmetric counter rotating eddies were formed, while at inclination angles greater than $0^{\circ}$, the symmetric flow and temperature patterns disappear.

Chan and Tien (1985a) investigated a square open cavity numerically, which had an isothermal vertical heated side facing the opening and two adjoining adiabatic horizontal sides. To obtain the satisfactory solutions in the open cavity the boundary conditions for field were approximated.

Chan and Tien (1985b) investigated shallow open cavities and made a comparison study using a square cavity in an enlarged computational domain. In the result they observed that for a square open cavity having an isothermal vertical side facing the opening and two adjoining adiabatic horizontal sides. Satisfactory heat transfer results could be obtained, especially at high Rayleigh numbers.

Mohammad (1995) investigated inclined open square cavities, by considering a restricted computational domain. The gradients of both velocity components were set to zero at the opening plane in that case which were different from those of Chan and Tien (1985a). In the result he found that heat transfer was not sensitive to inclination angle and the flow was unstable at high Rayleigh numbers and small inclination angles.

Rahman et al. (2009) investigated on Mixed Convection in a rectangular cavity with inlet, outlet and a heat conducting Horizontal Circular Cylinder. They found that both the heat transfer rate from the heated wall and the dimensionless temperature in the cavity strongly depends on the governing parameters and configurations of the system such as size, location, and thermal conductivity of the cylinder and location of the inflow, outflow opening.

\section{Ojectives}

To obtain numerical solution of the analytical model of the problem using finite element method. To visualize the fluid flow and temperature distribution inside the open cavity in terms of streamlines and isotherms respectively. To investigate the effects of $\mathrm{Gr}$ and $\mathrm{Pr}$ on the heat transfer parameter Nusselt number $(\mathrm{Nu})$ in an open cavity. To investigate the effects of inclination angles of the cavity on the heat transfer parameter $\mathrm{Nu}$ in the cavity.

To investigate the effects of high temperature of the bottom wall of the cavity and heat transfer at the cylinder. To develop a mathematical model regarding the effect of natural convection flow around a heated circular cylinder placed at the centre of a square open cavity.

\section{Research Methodology}

Finite Element (FE) analysis is a method to solve differential equations numerically which can be applied to many problems in engineering and scientific fields. Finite Element Simulation of natural convection in a two-dimensional rectangular open cavity has been studied. Top and bottom wall are kept at cool and hot temperature respectively.

This research starts from the two-dimensional NavierStoke's equations together with the energy equation to obtain the corresponding finite element equations. Galerkin's weighted Finite Element Method is applied to descretize the non-dimensional governing equations. Triangular Mesh is used to obtain the solution. Because this type of mesh can be used in any shape of domain, but rectangular mesh can be used in rectangular domain only.

\section{Model Specification}

The physical model considered here along with necessary geometric parameters. In this study heat transfer and the fluid flow in a two-dimensional square cavity of length L was considered, which is shown in the following schematic diagram.

\section{Mathematical Formulation}

The flow inside the cavity is assumed to be two-dimensional, steady, laminar, and incompressible and the fluid properties are to be constant. The radiation effects are taken 


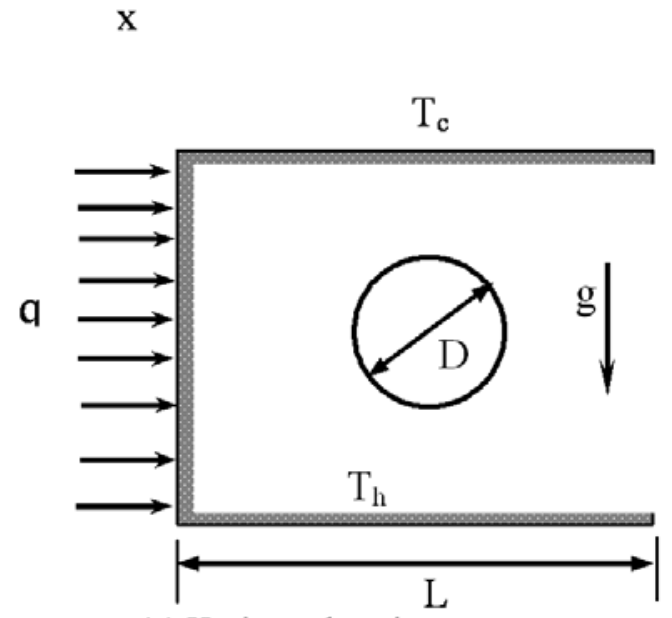

(a) Horizontal cavity

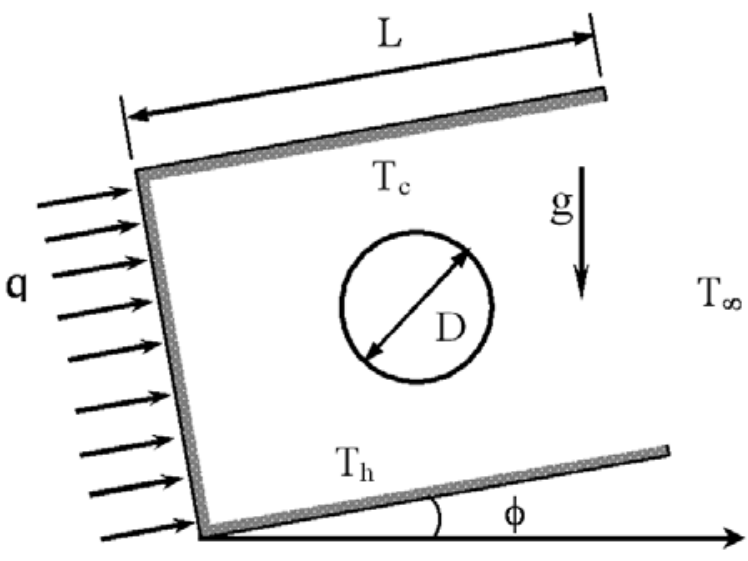

(b) Inclined cavity

Fig. 1: Schematic diagram of the physical problem

negligible and the Boussinessq approximation is used. The dimensionless governing equations describing the flow are as follows:

\section{Continuity equation}

\section{Momentum equations}

$$
\frac{\partial U}{\partial X}+\frac{\partial V}{\partial Y}=0
$$

$U \frac{\partial U}{\partial X}+V \frac{\partial U}{\partial Y}=-\frac{\partial P}{\partial X}+\frac{1}{\sqrt{G r}}\left(\frac{\partial^{2} U}{\partial X^{2}}+\frac{\partial^{2} U}{\partial Y^{2}}\right)+\theta \sin \phi$
$U \frac{\partial V}{\partial X}+V \frac{\partial V}{\partial Y}=-\frac{\partial P}{\partial Y}+\frac{1}{\sqrt{G r}}\left(\frac{\partial^{2} V}{\partial X^{2}}+\frac{\partial^{2} V}{\partial Y^{2}}\right)+\theta \cos \phi$

\section{Energy equation}

$U \frac{\partial \theta}{\partial X}+V \frac{\partial \theta}{\partial Y}=\frac{1}{\operatorname{Pr} \sqrt{G r}}\left(\frac{\partial^{2} \theta}{\partial X^{2}}+\frac{\partial^{2} \theta}{\partial Y^{2}}\right)$

\section{Boundary Conditons}

At Bottom wall: $U=V=0 ; \theta=1,0 \leq \mathrm{X} \leq 1$ and $\mathrm{Y}=0$

At Top wall: $U=V=0 ; \theta=0,0 \leq \mathrm{X} \leq 1$ and $\mathrm{Y}=1$

At the left wall : $\mathrm{U}=\mathrm{V}=0$; heat flux $\mathrm{q}=500, \mathrm{X}=0$, $0 \leq \mathrm{Y} \leq 1$

At the right side and open side: Convective Boundary Condition (CBC), $\mathrm{P}=0$.
Here the quantities $\mathrm{U}, \mathrm{V}$ are the non-dimensional velocity components of fluid; X,Y are non-dimensional Cartesian coordinates in the cavity; $\mathrm{q}$ is the constant heat flux from the left vertical wall of the cavity, $\phi$ is the inclinational angle of the cavity. Prandtl number Pr is the ratio of kinematic viscosity to the thermal diffusivity i.e. $\operatorname{Pr}=\frac{v}{\alpha}$, Grashof number Gr represents the ratio of the buoyancy force to the viscous force acting on the fluid and defined as

$$
\mathrm{Gr}=\frac{g \beta\left(T_{w}-T_{\infty}\right) L_{c}^{3}}{v^{2}}
$$

\section{Used Heat Transfer Parameter}

Since the flow field in natural convection is governed by the dimensionless

Grashof Number Gr. Where the Grashof number represents the ratio of the buoyancy force to the viscous force acting on the fluid and the reference velocity Uo is related to the buoyancy force term and is defined as

$$
U_{0}=\sqrt{g \beta L\left(T_{s}-T_{\infty}\right)}
$$

Here, $\mathrm{g}$ is the gravitational acceleration $\beta$, is volumetric thermal expansion coefficient , $1 / \mathrm{k} \quad\left(\beta=1 / \mathrm{T}\right.$ for ideal gases), $\mathrm{T}_{\mathrm{s}}$ is temperature at the surface, $\mathrm{T}_{\mathrm{O}}$ is the temperature of the fluid sufficiently far from the wall.

The Nusselt number $(\mathrm{Nu})$ is the non-dimensional heat transfer coefficient to be computed for heat transfer analysis in 
natural convection flow in the cavity. The Nusselt number for natural convection is a function of the Grashof number only. The local Nusselt number Nu can be obtained from the temperature field by applying the function

$$
N u=-\frac{1}{\theta(0, Y)}
$$

The overall or average Nusselt number was calculated by integrating the temperature gradient over the heated wall as follows:

$$
N u_{a v}=-\int_{0}^{1} \frac{1}{\theta(0, Y)} d y
$$

Since the dimensionless Prandtl number Pr is the ratio $\frac{v}{\alpha}$ of kinematic viscosity to thermal diffusivity. So $\operatorname{Pr}$ is a heat transfer parameter in the flow field of natural convection.

\section{Grid Independence Test}

To obtain grid independent solution, a grid refinement study is performed for a rectangular open cavity with $\mathrm{Gr}=10^{6}$ and $\mathrm{dr}=0.2$. Here diameter ratio $\mathrm{dr}$ is the ratio of diameter of the cylinder and length of the cavity. The test shows the convergence of the average Nusselt number $\mathrm{Nu}$ at the heated surface with grid refinement. It is observed that grid independence is achieved with 13686 elements where there is insignificant change in $\mathrm{Nu}$ with further increase of mesh elements. The grid refinement tests are done by taking six different non-uniform grids with the following number of nodes and elements: 27342 nodes, 4818 elements; 49335 nodes, 7663 elements; 72782 nodes, 10365 elements; 73542 nodes, 11413 elements; 96030 nodes, 12356 elements; 892450 nodes, 13686 elements. In this case 982450 nodes, 13686 elements can be chosen through the processing to optimize the relation between the accuracy required and the computational time.

\section{Code Validation}

Due to lack of availability of experimental and numerical results published earlier on this regard, validation of this prediction could not be done. The present numerical results average Nusselt numbers Nuav are verified in Table I against documented numerical study, namely; Finite element simulation of natural convection from an open rectangular cavity containing adiabatic circular cylinder the M. Phil. thesis by Koabra (2008) BUET, Dhaka.
Table I: Results for the different Grashof numbers with $\operatorname{Pr}=0.72, \mathrm{dr}=0.2$

\begin{tabular}{l|c|c}
\hline \multirow{2}{*}{$G r$} & \multicolumn{2}{|c}{$\mathrm{Nu}_{\mathrm{av}}$} \\
\cline { 2 - 3 } & Present work & Koabra (2008) \\
\hline $10^{3}$ & 3.3282 & 3.2473 \\
$10^{4}$ & 3.4014 & 3.3020 \\
$10^{5}$ & 4.4181 & 4.1825 \\
$10^{6}$ & 5.8227 & 5.5521 \\
\hline
\end{tabular}

\section{Results and Discussions}

The results Nuav are obtained for Grashof number from $10^{3}$ to $10^{6}$ at $\operatorname{Pr}=0.72,1.0,7.0$ while inclination angles $0^{\circ}$ to $45^{\circ}$ and different high temperatures $320^{\circ} \mathrm{k}, 350^{\circ} \mathrm{k}, 375^{\circ} \mathrm{k}$ at bottom wall with constant physical properties. Here the parametric analysis for a wide range of governing parameters shows consistence performance of the present numerical approach to obtain as streamlines and temperature profiles. The obtained results show that the heat transfer coefficient $\mathrm{Nu}$ is strongly affected by Grashof number. An empirical correlation can be developed using Nusselt number and Grashof number.

In the effect of Grashof number, the isotherm figures show that as the Grashof number and the inclination angle increases the buoyancy force increases and the thermal boundary layers become thinner. In Fig. 2 streamlines patterns for $\operatorname{Pr}=$ $0.72,1.0,7.0$, high temperature $\mathrm{T}_{\mathrm{h}}=320^{\circ} \mathrm{k}$ at the bottom wall, low temperature $\mathrm{T}_{1}=276^{\circ} \mathrm{k}$ at the upper wall and $\mathrm{T}_{c l}=300 \mathrm{ok}$ temperature of the cylinder show that the fluid moves from the bottom wall of the cavity, circulates in a clockwise direction around the cylinder making the shape of the cavity and moves toward the upper part of the cavity and fresh cool fluid enter the cavity through the bottom wall. The isotherm patterns are shown in Fig. 3. The streamlines patterns are very similar for different inclinations while $\mathrm{Gr}=$ $10^{3}$. The fluid moves faster for $\mathrm{G}=10^{4}$ to $10^{6}$ and the upper boundary layer becomes thinner. One partial vortex is seen at the centre of the opening side for each of $\mathrm{Gr}=10^{3}$ to $10^{6}$, because of the effect of the heated circular cylinder. One vortex is formed near the cylinder for each of $\mathrm{Gr}=10^{4}, 10^{5}$ and two vortices are formed for $\mathrm{Gr}=10^{6}$ near the bottom wall. Streamlines show that as the inclination angle of the heated wall increases, the velocity gradient remained almost same as before for $\operatorname{Pr}=0.72,1$ and 7.0. The variations of the average Nusselt number and the average temperature are also presented. The results are obtained for a Grashof numbers ranging from $10^{3}$ to $10^{6}$ and for inclination angles range 


$$
\mathrm{Gr}=10^{3}
$$

$\mathrm{Gr}=10^{4}$

$\mathrm{Gr}=10^{5}$

$\mathrm{Gr}=10^{6}$
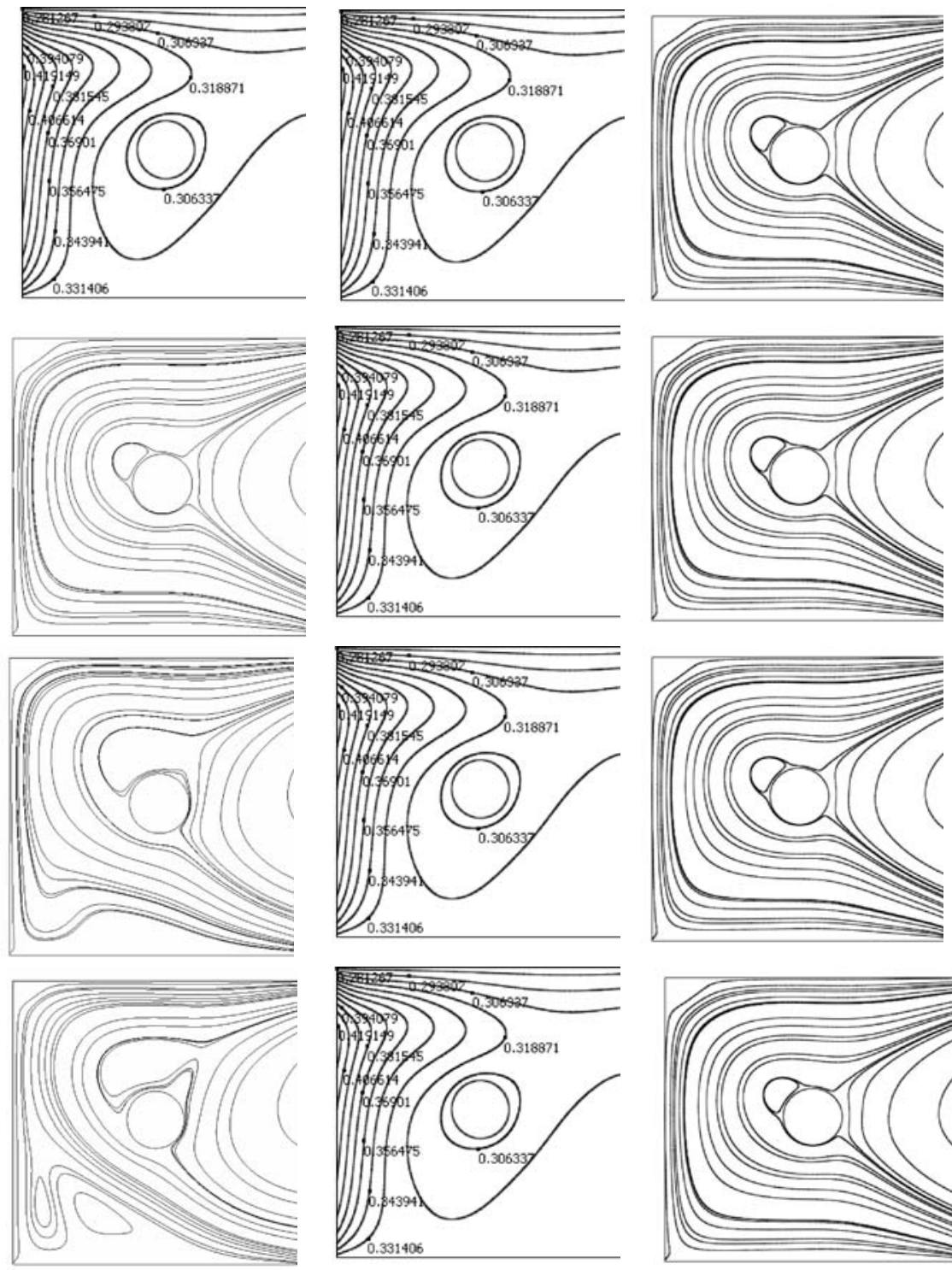

$\operatorname{Pr}=0.72, \phi=0^{\circ}$

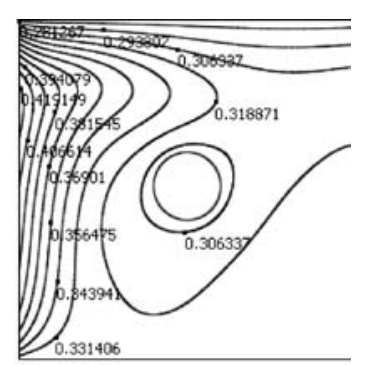

$\operatorname{Pr}=1.0, \phi=30^{\circ}$
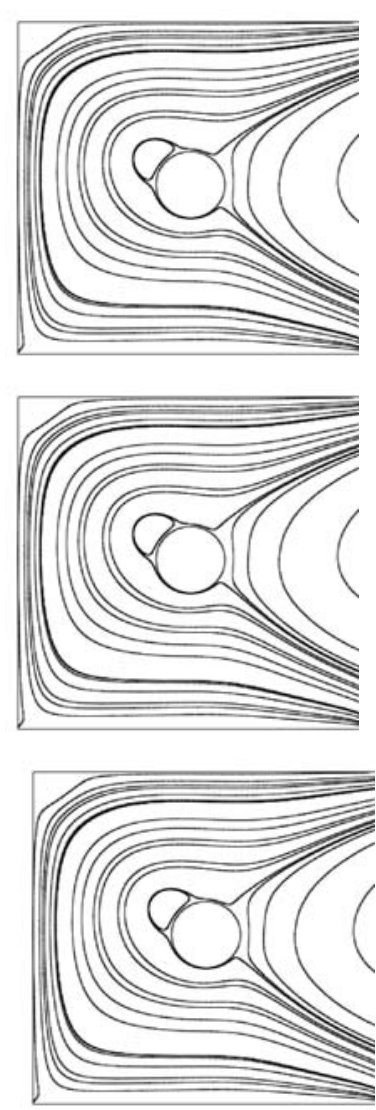

$\operatorname{Pr}=7.0, \phi=45^{\circ}$

Fig. 2 Streamlines for $\mathrm{dr}=0.2, \mathrm{Gr}=10^{3}, 10^{4}, 10^{5}, 10^{6} \& \mathrm{Th}=320^{\circ} \mathrm{k}$

from $0^{\circ}$ to $45^{\circ}$ of the cavity. In figures of streamlines are almost laminar for $\mathrm{Gr}=10^{3}$ while $\mathrm{Pr}=7.0$. Due to the effect of the heated cylinder, a partial vortex is seen the open side of the cavity for each of $\mathrm{Gr}=10^{3}$ to $10^{6}$. Due to increase in $\mathrm{T}_{\mathrm{h}}$ at the bottom wall, the fluid rises faster from the bottom wall and one vortex is created for each of $\mathrm{Gr}=10^{4}, 10^{5}$ near the cylinder, one small vortex is formed near the bottom wall while $\mathrm{Gr}=10^{5}$. Two vortices are formed near the bottom wall for $\mathrm{Gr}=10^{6}$. The streamlines and isotherms for $\mathrm{dr}=0.2$, $\operatorname{Pr}=1.0, \mathrm{~T}_{\mathrm{h}}=375^{\circ} \mathrm{K}, \mathrm{T}_{1}==278^{\circ} \mathrm{K}$ and for inclination angles $\phi=0^{\circ}, 15^{\circ}, 30^{\circ}, 45^{\circ}$ are shown in Figs. $6 \& 7$ respectively for the variation of Grashof number. Effects of average Mussels number against Grashof number at the cylinder for different inclination angle of the cavity are shown in the figures Fig. 4, Fig.5 and Fig. 8. In Fig. 4, Fig. 5 and Fig. 8 the investigation found that the average $\mathrm{Nu}_{\mathrm{av}}$ at the cylinder is large while inclination angle $\phi=0^{\circ}$ and gradually decreases for increase in $\phi$ up to $45^{\circ}$. Because when $\phi=0^{\circ}$ the upper side of the cavity is just above the cylinder and due to greater temperature difference more heat transfer occurs at this position. But when the cavity inclined at angles $15^{\circ}, 30^{\circ}, 45^{\circ}$ etc the top wall of the cavity tilted left to the cylinder gradually; the open side of the cavity become just above the cylinder gradually and at this position the temperature difference between 

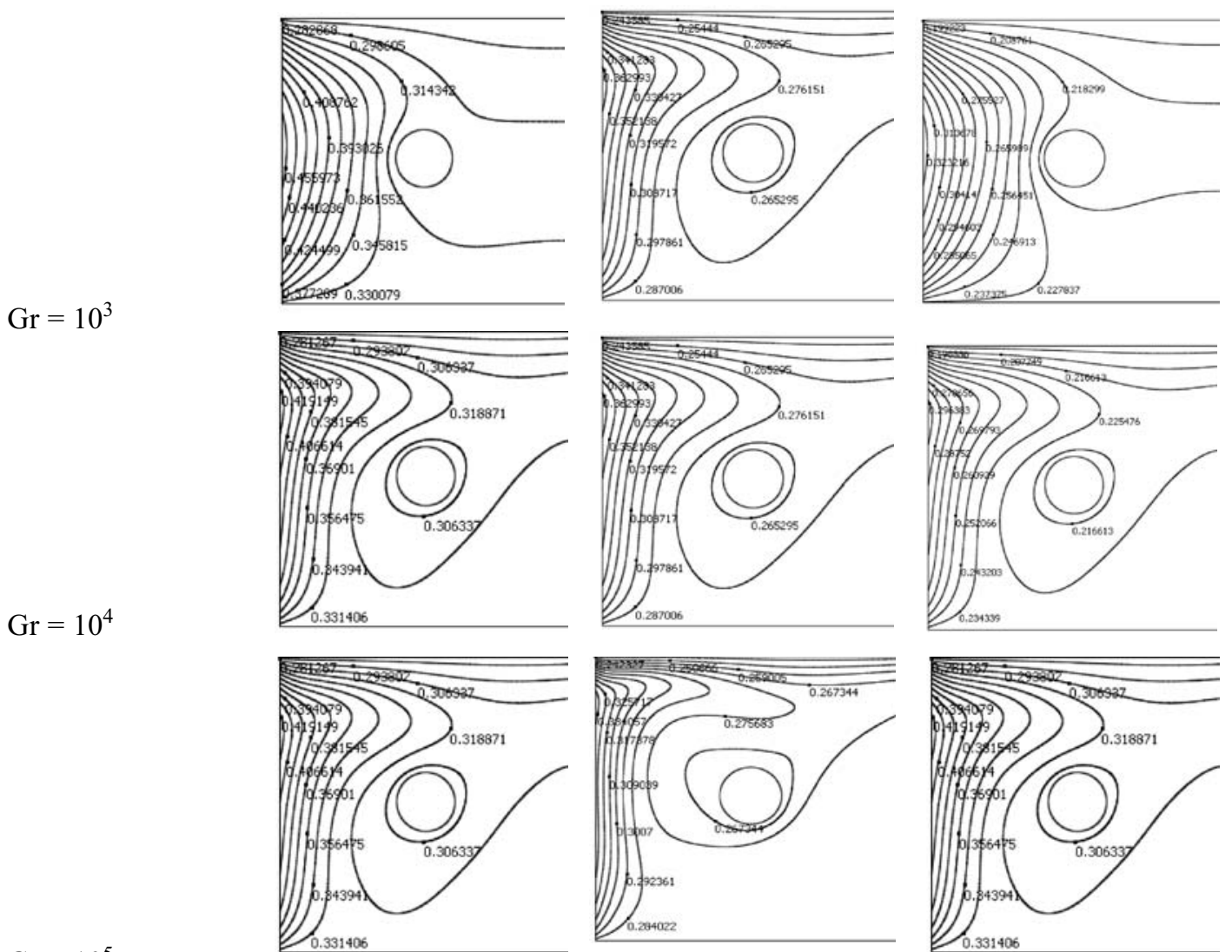

$\mathrm{Gr}=10^{5}$
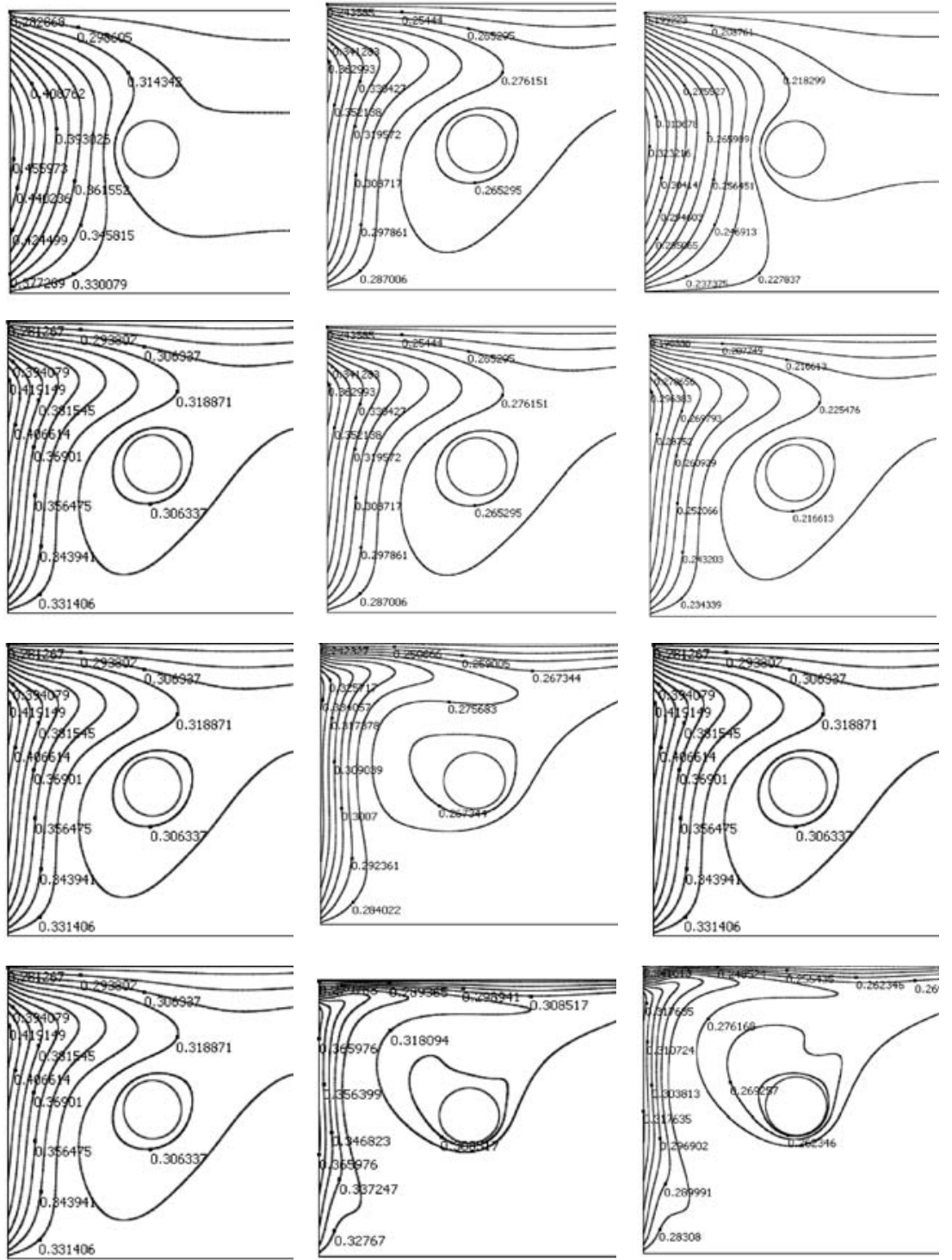

$\mathrm{Gr}=10^{6}$

$\operatorname{Pr}=0.72, \Phi=0^{\circ}$

$\operatorname{Pr}=1.0, \Phi=30^{\circ}$

$\operatorname{Pr}=7, \Phi=45^{\circ}$

Fig 3: Figures of Isotherm's for $\mathrm{dr}=0.2, \mathrm{Gr}=10^{3}, 10^{4}, 10^{5}, 10^{6} \& \mathrm{~T}_{\mathrm{h}}=320$

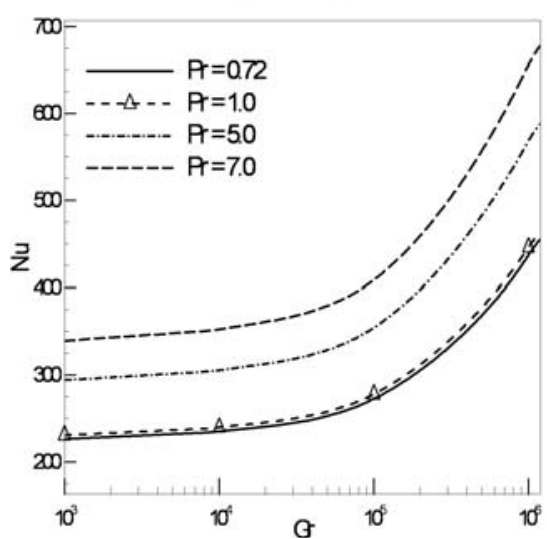

Fig. 4: Variation of average Nusselt number against Gr for different inclination angles while $\mathrm{Pr}=\mathbf{0 . 7 2}, \mathrm{dr}$ $=0.2$ at the cylinder.

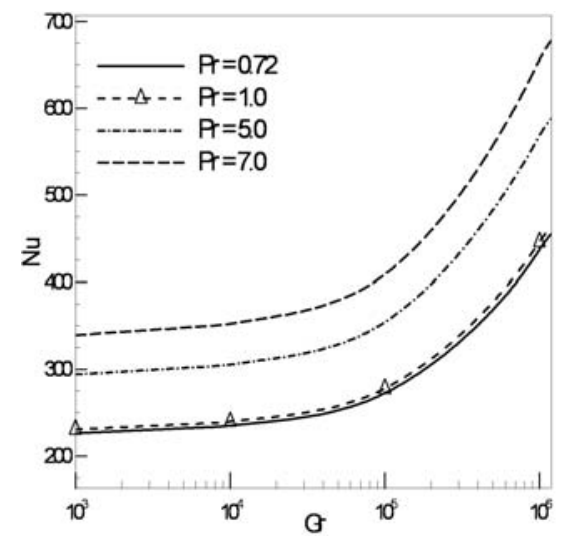

Fig. 5: Variation of average Nusselt number against Gr for different inclination angles while $\mathrm{Pr}=1.0, \mathrm{dr}$ $=0.2$ at the cylinder. 
$\mathrm{Gr}=10^{3}$
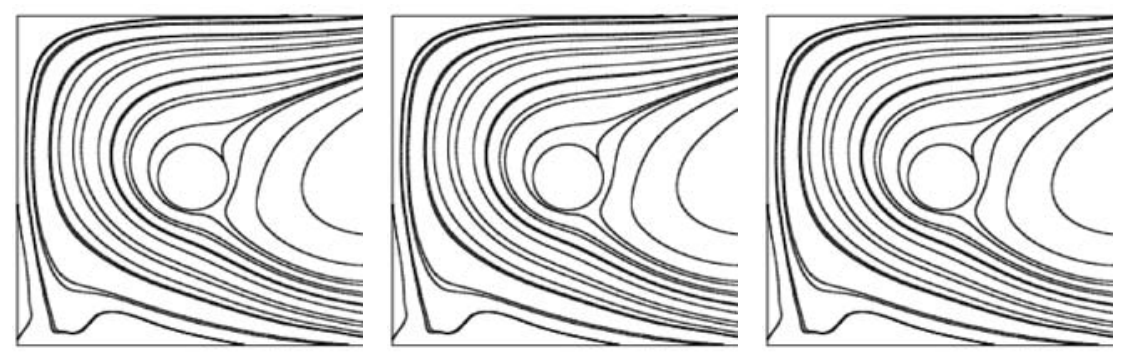

$\mathrm{Gr}=10^{4}$
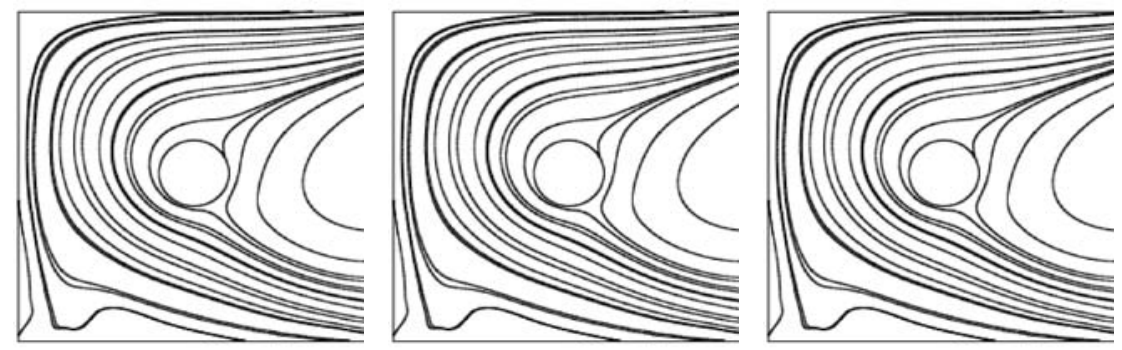

$\mathrm{Gr}=10^{5}$
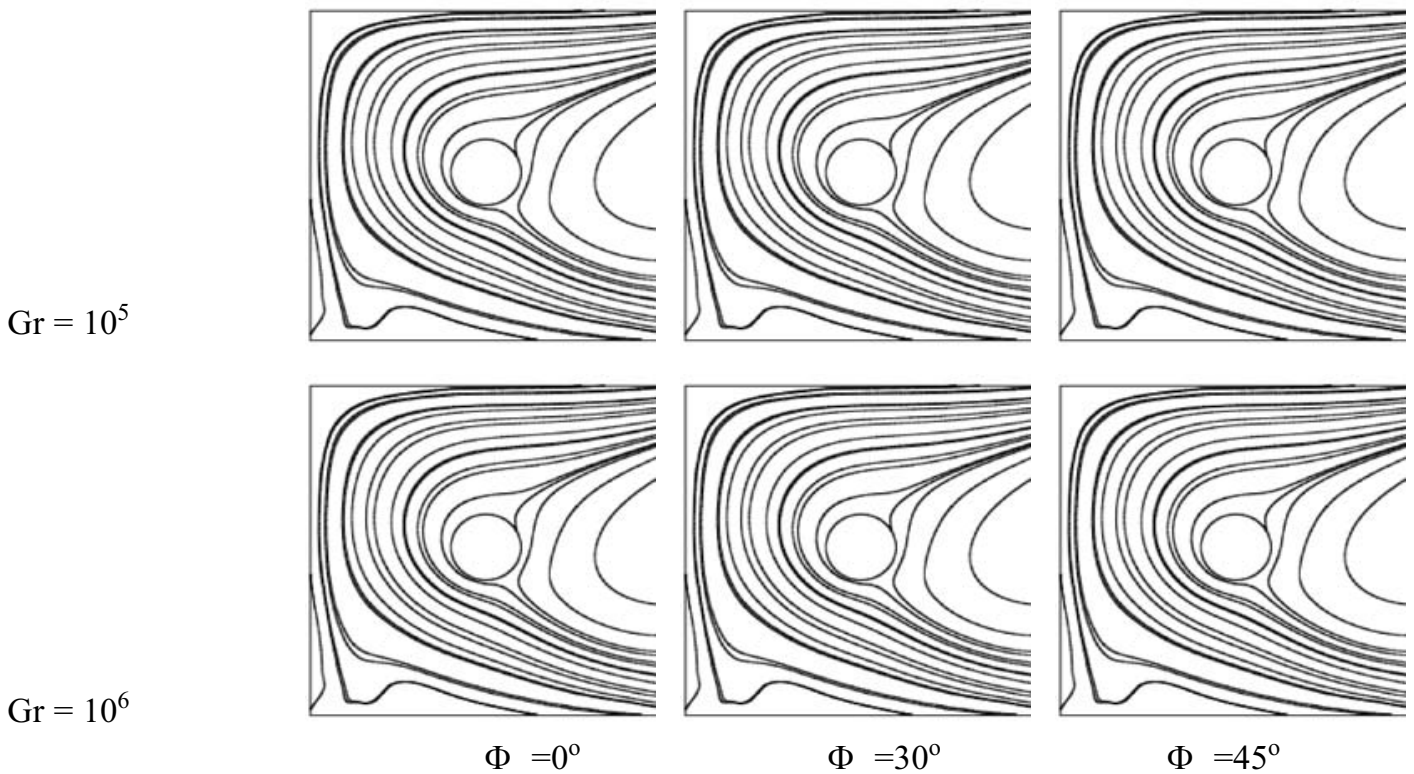

$\Phi=30^{\circ}$

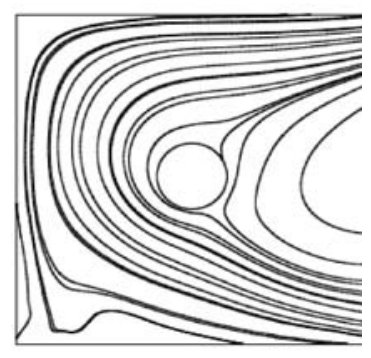

$\Phi=45^{\circ}$
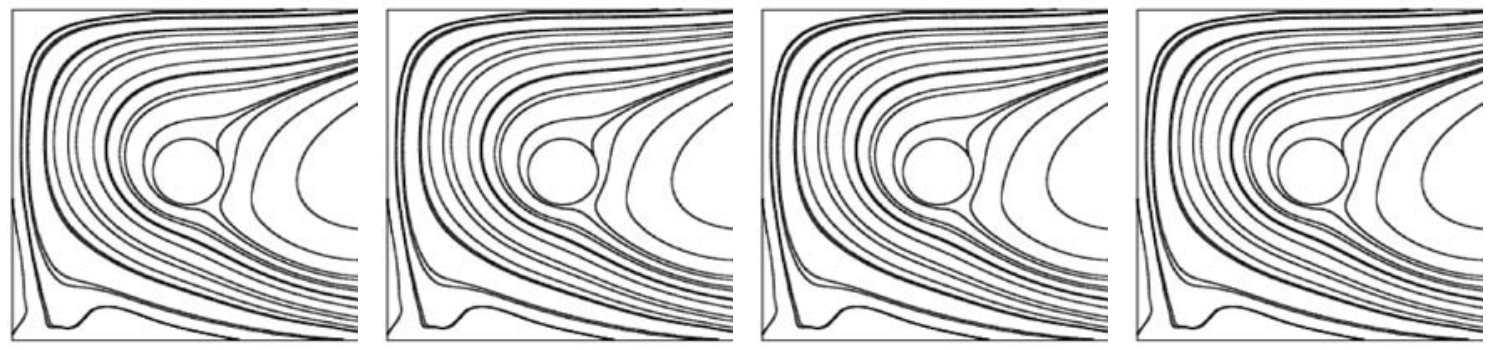

$\mathrm{Gr}=10^{3} \quad \Phi=15^{\circ} \quad \mathrm{Gr}=10^{4} \quad \Phi=15^{\circ}$

$\mathrm{Gr}=10^{5} \quad \Phi=15^{\circ}$

$\mathrm{Gr}=10^{6} \quad \Phi=15^{\circ}$

Fig.6.Streamlines patterns for $\mathrm{dr}=0.2, \mathrm{Th}=375^{\circ} \mathrm{k}, \mathrm{Pr}=1.0$ 

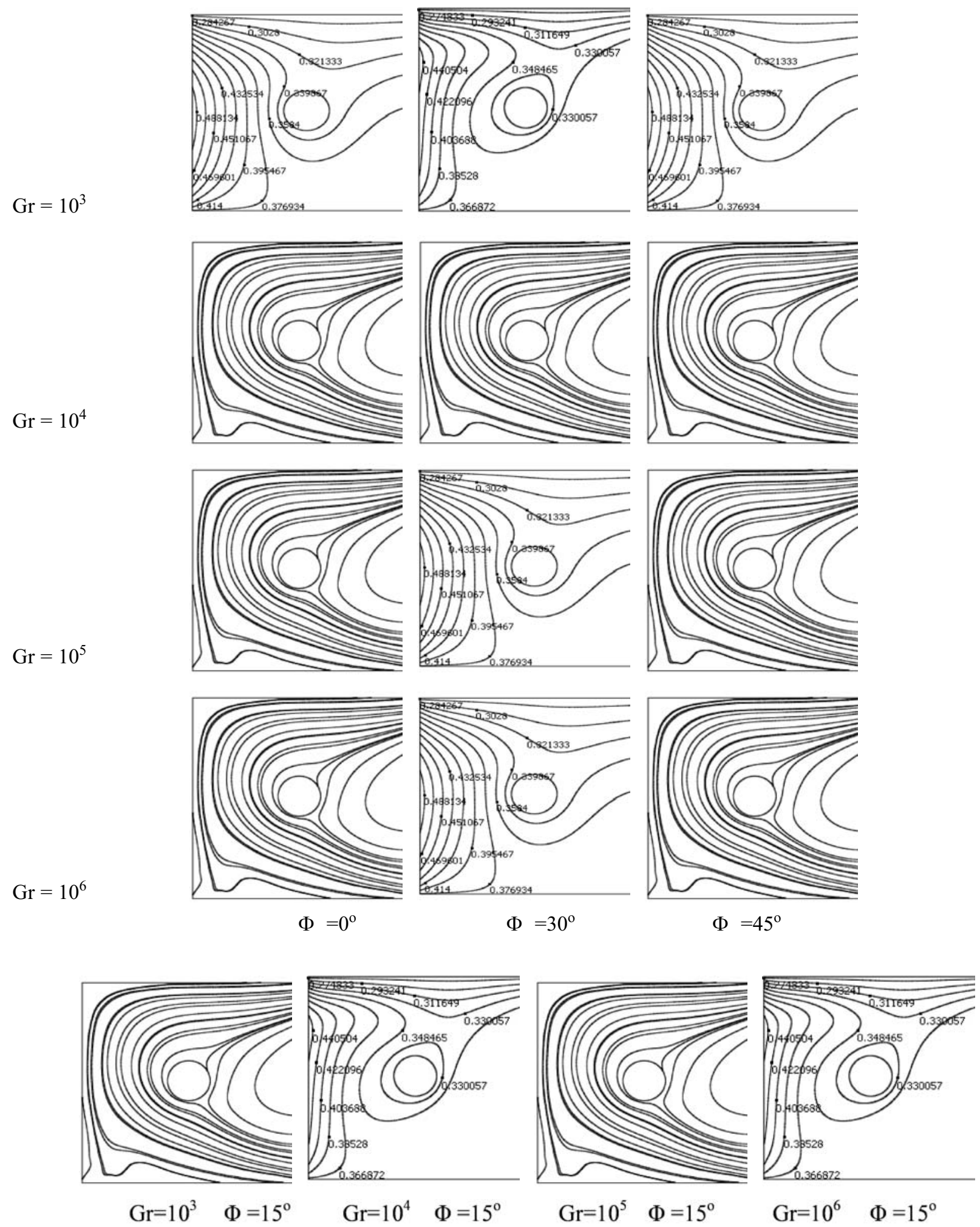

Fig.7. Isotherm patterns for $\mathrm{dr}=0.2, \operatorname{Pr}=1.0, \mathrm{Th}=375^{\circ} \mathrm{k}$ 
Table II: Average Nusselt number $\mathrm{Nu}_{\mathrm{av}}$ for different inclination angles $\phi$ and Grashof numbers with $\operatorname{Pr}=0.72$ and $d r=0.2$.

\begin{tabular}{l|c|c|c|c}
\hline$\phi$ & \multicolumn{4}{|c}{$\mathrm{Nu}_{\mathrm{av}}$} \\
\cline { 2 - 5 } & $\mathrm{Gr}=10^{3}$ & $\mathrm{Gr}=10^{4}$ & $\mathrm{Gr}=10^{5}$ & $\mathrm{Gr}=10^{6}$ \\
\hline $0^{\mathrm{o}}$ & $3 . .2725$ & $3 . .29$ & 4.07 & 5.4947 \\
$15^{\mathrm{o}}$ & $3 . .2756$ & $3 . .37$ & 4.2837 & $5 . .8547$ \\
$30^{\circ}$ & $3 . .3525$ & $3 . .5443$ & 4.5646 & $5 . .9657$ \\
$45^{\circ}$ & 3.4125 & 3.4956 & 4.7543 & $5 . .9757$ \\
\hline
\end{tabular}

the cylinder and the ambient temperature $T_{\infty}$ is less than the previous position. Because of this, the heat transfer at the cylinder decreases at the higher inclination angles gradually. In Fig. 9 variation of average Nusselt numbers at the cylinder against Gr for different $\operatorname{Pr}$ while $\phi=0^{\circ}$ are shown graphically. In this figure $\mathrm{Nu}_{\mathrm{av}}$ at the cylinder is lowest for $\operatorname{Pr}=$ 0.72. $\mathrm{Nu}_{\mathrm{av}}$ increases for $\mathrm{Pr}=1.0,5.0,7.0$ and for increase in $\mathrm{Gr}$. i.e. $\mathrm{Nu}$ is an increasing function of $\mathrm{Pr}$ and $\mathrm{Gr}$.

One vortex is created for each of $\mathrm{Gr}=10^{3}, 10^{4}, 10^{5}, 10^{6}$ on the open side. This unsteady streamlines and isotherms are occurred due to higher Prandtl number $\operatorname{Pr}=7.0$. Since the relation between $\mathrm{Nu}$ and $\mathrm{Pr}$ is non-linear and one vortex is formed near the bottom wall for $\mathrm{Gr}=10^{6}$. The isotherms patterns of these figures are similar.

The streamlines and isotherms for $\mathrm{dr}=0.2, \operatorname{Pr}=1.0, \mathrm{~T}_{\mathrm{h}}=$ $375^{\circ} \mathrm{k}$ and for inclination angles $\phi=0^{\circ}, 15^{\circ}, 30^{\circ}, 45^{\circ}$ are shown in Fig. $6 \&$ Fig. 7 respectively for the variation of

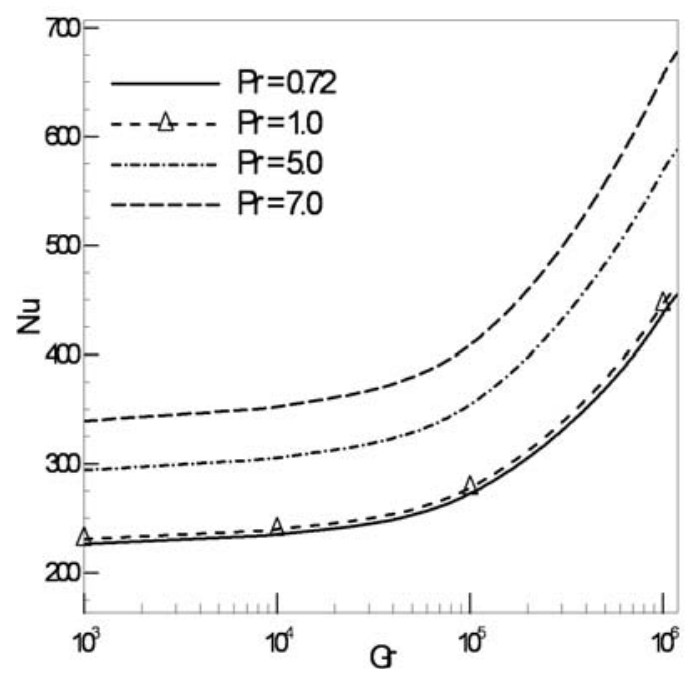

Fig. 8: Variation of average Nusselt number against Gr for different inclination angles while $\mathrm{Pr}=$ $7.0, d r=0.2$ at the cylinder.
Table III: Average Nusselt numbers $\mathrm{Nu}_{\mathrm{av}}$ for different Pr. and Gr taking $P r=0.72,1.0$ and 7.0, angle $\phi=0^{\circ}$ and $\mathrm{dr}=0.2$

\begin{tabular}{l|c|c|c|c}
\hline \multirow{2}{*}{$\operatorname{Pr}$} & \multicolumn{4}{|c}{$\mathrm{Nu}_{\mathrm{av}}$} \\
\cline { 2 - 5 } & $\mathrm{Gr}=10^{3}$ & $\mathrm{Gr}=10^{4}$ & $\mathrm{Gr}=10^{5}$ & $\mathrm{Gr}=10^{6}$ \\
\hline 0.72 & 3.2825 & 3.2975 & 4.2575 & 5.6747 \\
1.0 & 3.2734 & 3.3595 & 4.3465 & 5.8857 \\
7.0 & 3.3255 & 4.4231 & 4.8753 & 8.8447 \\
\hline
\end{tabular}

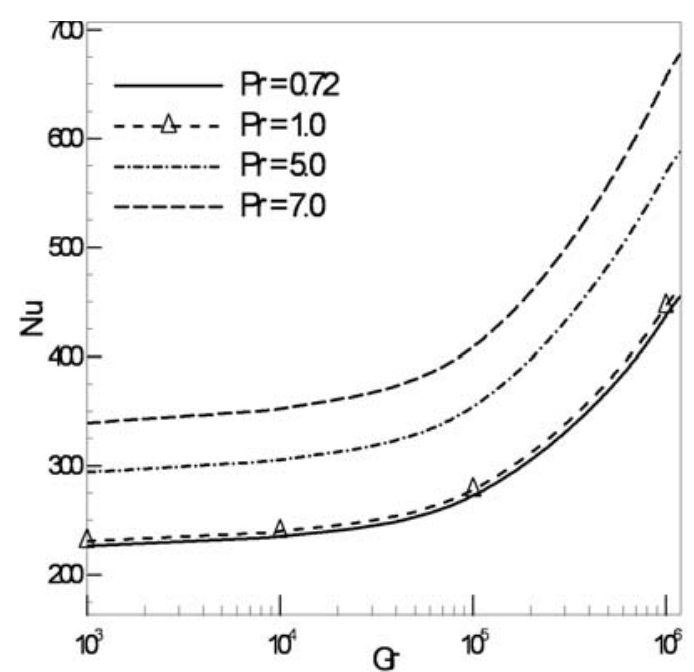

Fig. 9: Variation of average Nusselt number against Gr for different Prandtl numbers while $\phi=0$, $d r=0.2$. at the cylinder.

Grashof number. For Grashof number $10^{3}$, the streamlines are almost laminar but one vortex is created for each of $\mathrm{Gr}=$ $10^{4}, 10^{5}$; but two recirculation cells are formed near the cylinder and bottom wall for $\mathrm{Gr}=10^{6}$. One small vortex is formed at the bottom wall. One vortex is created near the cylinder for each of $\mathrm{Gr}=10^{3}, 10^{4}$ Two recirculations are formed for $\mathrm{Gr}=10^{5}, 10^{6}$ one of these is near the cylinder and another is near the bottom wall. This is due to increase in temperature at the bottom wall and presence of the highly heated circular cylinder and cylinder redirect the fluid.

\section{Conclusion}

The following characteristics are found in the research work.

* Heat transfer rate depends on Prandtl number and heat transfer rate increases as Prandtl number increases. 
* Thermal boundary layer thickness becomes thinner for higher Grashof number.

* The heat transfer rate decreases for $\mathrm{Gr}=10^{3}$ and increases gradually for increasing of Grashof number.

* Various vortices are formed in the flow field in the cavity for higher Grashof numbers.

* The heat transfer rate $\mathrm{Nu}$ increases at the cylinder as inclination angle of the cavity increases and increases of Grashof number.

\section{Acknowledgments}

We acknowledge the computing facilities in the computer lab of the Department of Mathematics of Bangladesh University of Engineering and Technology Dhaka. We would like to extend our thanks and gratitude to honorable reviewer of BJSIR for his valuable remarks on our work.

\section{References}

ChanY. L. and Tien C. L (1985b). A Numerical study of twodimensional natural convection in square open cavities, Numerical Heat Transfer, 8: 65-80.

ChanY. L. and Tien C. L. (1985a). A Numerical study of twodimensional laminar natural convection in a shallow open cavity, Int. J. Heat Mass Transfer, 28: 603-612.
Fariza Tul Koabra (2008). Finite Element Simulation of Natural Convection from an open rectangular Cavity containing Adiabatic Circular Cylinder. [M. Phil. thesis in Mathematics], BUET, Dhaka, Bangladesh.

Mohamad A, (1995). Natural convection in open cavities and slots. Numerical Heat Transfer, 27: 705-716.

Rahman Md. M., Alim M. A. and Mamun M. A H. (2009). Finite Element Analysis of Mixed Convection in a Rectangular Cavity with a Heat -Conducting Horizontal Circular Cylinder, Journal of Non Linear Analysis: Modelling and Control, 14: No 2.

Showole R. A. and Tarasuk J. D (1993). Experimental and numerical studies of natural convection with flow separation in upward-facing inclined open cavities, Journal of Heat Transfer, 115: 592-605.

Received : February 10, 2011,

Accepted : July 21, 2011. 